hep-th/0002117

EFI-2000-4

\title{
D-Branes as Unstable Lumps in Bosonic Open String Field Theory
}

\author{
Jeffrey A. Harvey and Per Kraus \\ Enrico Fermi Institute and Department of Physics \\ University of Chicago, Chicago, IL 60637, USA \\ harvey, pkraus@theory.uchicago.edu
}

\begin{abstract}
We construct Dp-branes in bosonic string theory as unstable lumps in a truncated string field theory of open strings on a D25-brane. We find that the lowest level truncation gives good quantitative agreement with the predicted D-brane tension and low-lying spectrum of the D-brane for sufficiently large $p$ and study the effect of the next level corrections for $p=24$. We show that a $U(1)$ gauge field zero mode on the $\mathrm{D}$-brane arises through a mechanism reminiscent of the Randall-Sundrum mechanism for gravity.
\end{abstract}

February, 2000 


\section{Introduction}

In a remarkable paper, Kostelecky and Samuel studied tachyon condensation in Witten's version of bosonic open string field theory [1] using a level truncation scheme and found what appears to be rapid convergence as higher levels are included [2]. This calculation was recently reinterpreted and extended in [3]. Following previous arguments [4], Sen and Zwiebach argued that this calculation describes the decay of a space filling D25-brane to the bosonic string vacuum and found that the energy of tachyon condensation cancels the $D 25$-brane tension to an accuracy of $99 \%$ when terms in the tachyon potential up to level 8 are included. The level truncation scheme has been further extended in [5] and tachyon condensation has been studied to lowest order in open superstring field theory in [6].

Based on studies of tachyon condensation in conformal field theory [7], one also expects to be able to describe $D p$-branes for $p<25$ as unstable configurations of the open string tachyon on a space-filling $D 25$-brane $[8]$. It has been suggested that the level truncation scheme might be a useful tool for studying this question [3]. Such an analysis probes much more of the structure of open string field theory than a study of the tachyon vacuum energy since it involves the higher derivative terms present in string field theory in a non-trivial way.

In this paper we begin an investigation of $D p$-branes in level truncated open string field theory. We find that for $p=24$ the lowest level terms give a remarkably good description of both the qualitative and quantitative structure of the D24-brane. For smaller $p$, higher derivative terms and higher level fields become progressively more important and require a more detailed analysis than will be presented here.

\section{2. $D p$-branes at level 0}

We will follow the conventions of [2]. For convenience we set $\alpha^{\prime}=1, g=2$, as in [3] with $g$ the open string coupling constant. In these conventions a $D p$-brane has tension

$$
T_{p}=\frac{1}{2}(2 \pi)^{23-p}=(2 \pi)^{25-p} T_{25} .
$$

The spectrum of open strings on the $D$-brane includes a tachyon with $m^{2}=-1$, a massless $U(1)$ gauge field, massless scalars corresponding to translational zero modes, and additional massive states. 
Kostelecky and Samuel used open string field theory to work out the D25-brane action to the first few levels. Truncating at level 0 leaves just the tachyon $\phi$ with action

$$
S=2 \pi^{2} T_{25} \int d^{26} x\left(\frac{1}{2} \partial_{\mu} \phi \partial^{\mu} \phi-\frac{1}{2} \phi^{2}+2 \kappa \tilde{\phi}^{3}\right),
$$

where

$$
\kappa=\frac{1}{3 !}\left(\frac{3 \sqrt{3}}{4}\right)^{3}, \quad \tilde{\phi}=\exp \left[\ln (3 \sqrt{3} / 4) \partial_{\mu} \partial^{\mu}\right] \phi .
$$

We are using a metric with signature $\eta_{\mu \nu}=\operatorname{diag}(-,+,+, \ldots,+)$. In the remainder of this section we will set $\tilde{\phi}=\phi$, and defer discussion of the validity of this substitution to the next section.

The tachyon potential $V(\phi)=-\frac{1}{2} \phi^{2}+2 \kappa \phi^{3}$ has an unstable extremum at $\phi=0$ and a locally stable extremum at $\phi_{c}=1 /(6 \kappa) \approx .456$. The local minimum $\phi_{c}$ is unstable due to nucleation of bubbles in which the tachyon is sufficiently negative, $\phi<\phi_{*}=-\phi_{c} / 2$, where $V\left(\phi_{*}\right)=V\left(\phi_{c}\right)$. The nucleation of a bubble is described by a "bounce" — a solution of the Euclidean field equations which asymptotes to $\phi_{c}$. Such a bounce solution has a single negative mode in its fluctuation spectrum. Since the action scales like $1 / g^{2}$, there is a natural candidate for the bounce: the bosonic $D$-instanton. This situation is to be contrasted with that in IIA string theory, which also contains a $D$-instanton with a single negative mode. The IIA D-instanton does not mediate vacuum decay, but instead signals the existence of a non-contractible loop in the space of Euclidean IIA histories [9].

Besides the D-instanton, the lowest order tachyon action supports various $p+1$ dimensional unstable "lump" solutions, and it has been suggested that these should be identified with bosonic $D p$-branes [8]. Consider the field equations for static configurations with $p+1$ dimensional translation symmetry. Let $x^{m}, m=0 \ldots p$, be coordinates parallel to the lump, and let $\rho$ be the radial coordinate in the $25-p$ dimensional transverse space. Then the equation of motion for spherically symmetric tachyon configurations is given by

$$
\partial_{\rho}^{2} \phi+\frac{24-p}{\rho} \partial_{\rho} \phi-V^{\prime}(\phi)=0
$$

If we think of $\rho$ as time, then (2.4) can be thought of as the equation of motion for a particle in a potential $-V$ subject to a time dependent damping term (which vanishes for $p=24)$.

A lump has boundary conditions

$$
\left.\partial_{\rho} \phi\right|_{\rho=0}=0, \quad \lim _{\rho \rightarrow \infty} \phi(\rho)=\phi_{c} .
$$


For $p<24, \rho$ ranges from 0 to $\infty$ and smoothness at the origin requires that the $\rho$ derivative of $\phi$ vanish there. For $p=24, \rho$ ranges from $-\infty$ to $\infty$ and the vanishing derivative at the origin follows from the $\rho \rightarrow-\rho$ symmetry.

For $p=24$, translation symmetry implies the conservation law

$$
\frac{1}{2}\left(\partial_{\rho} \phi\right)^{2}-V(\phi)=-V\left(\phi_{c}\right)=\frac{1}{6^{3} \kappa^{2}},
$$

with solution given by

$$
\rho=\int_{\phi_{*}}^{\phi} \frac{d \phi^{\prime}}{\sqrt{2 V\left(\phi^{\prime}\right)-2 V\left(\phi_{c}\right)}} .
$$

It does not seem possible to express $\phi(\rho)$ in closed form. Numerical evaluation yields the form shown by the solid line in figure 1 .

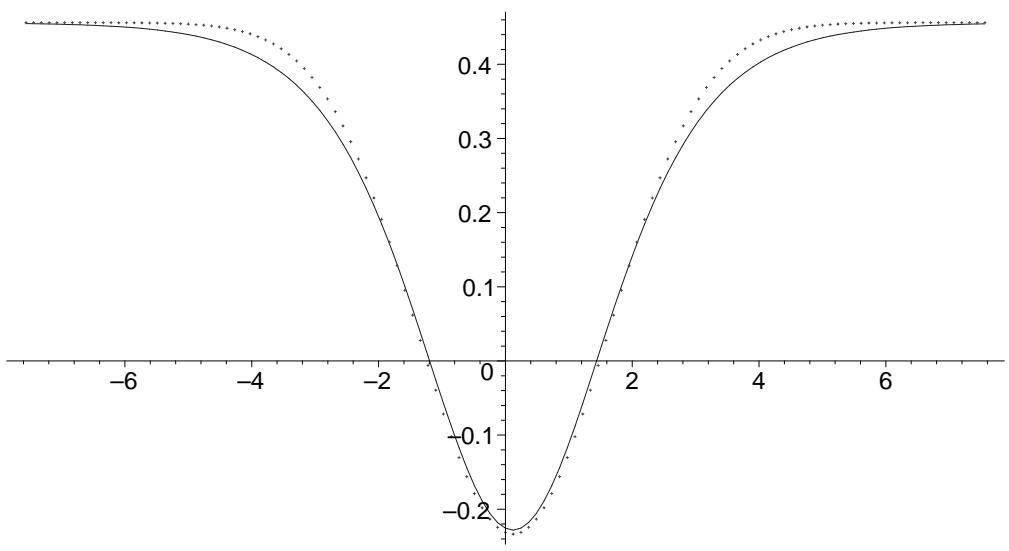

Fig. 1: The lump solution, plotted as $\phi$ versus $\rho$. The solid line is the numerical solution, and the dashed line represents the approximate fit $\phi_{\ell}$.

The exact lump solution is well approximated by the function

$$
\phi_{\ell}(\rho)=\phi_{c}-.69 e^{-.22 \rho^{2}},
$$

as illustrated by the dashed line in figure 1 . We will often find it convenient to work with $\phi_{\ell}(\rho)$, which suffices for the accuracy needed in this paper.

The tension of the lump is given by

$$
\begin{aligned}
T_{24}^{\ell} & =2 \pi^{2} T_{25} \int_{-\infty}^{\infty} d \rho\left\{\frac{1}{2}\left(\partial_{\rho} \phi\right)^{2}+V(\phi)-V\left(\phi_{c}\right)\right\} \\
& =4 \pi^{2} T_{25} \int_{\phi_{*}}^{\phi_{c}} d \phi^{\prime} \sqrt{2 V\left(\phi^{\prime}\right)-2 V\left(\phi_{c}\right)} \approx 4.93 T_{25} \approx .78 T_{24} .
\end{aligned}
$$


We thus find that $T_{24}^{\ell}$ is $78 \%$ of $T_{24}$, which supports the conjecture that the lump represents a $D 24$-brane.

Now we turn to the description of $D p$-branes with $p<24$. Here we have to proceed numerically. We use the shooting method, which corresponds to tuning $\phi(0)$ such that the solution asymptotes smoothly to $\phi_{c}$ at infinity. We find the following results for $\phi(0)$ and the tension $T_{p}^{\ell}$ expressed in terms of the $D p$-brane tension $T_{p}$

\begin{tabular}{|c|r|r|r|r|r|r|r|}
\hline $\mathrm{p}$ & 24 & 23 & 22 & 21 & 20 & 19 & $<19$ \\
\hline$\phi(0)$ & -.23 & -.64 & -1.5 & -3.5 & -11.5 & $-10^{7}$ & no sol. \\
\hline$T_{p}^{\ell} / T_{p}$ & .78 & .81 & .72 & .59 & .30 & .003 & no sol. \\
\hline
\end{tabular}

The accuracy rapidly decreases with decreasing $p$. For $p<19$ we find no solutions; the damping term in the equation of motion prevents the field from making it over the hump no matter how high up the inverted potential we take the field at the origin. As the magnitude of $\phi(0)$ increases, the solutions develop a region of increasing $\partial_{\rho} \phi$ near the origin. Thus the approximation of neglecting higher derivatives and higher level fields becomes worse and worse, which accounts for the decreasing accuracy of the computed tension.

\section{Corrections to the D24-brane tension}

Surprisingly, truncation to the lowest level fields and lowest number of derivatives gives good agreement for the tension of $D p$-branes for sufficiently large $p$. There is no a priori reason why this should be the case, nor is it clear that including higher levels and higher derivatives will yield small corrections to the lowest order result. However, [2,3] found that this was the case for the value of the minimum of the tachyon potential (although in this case higher derivatives played no role) and so we might hope that the same is true here. We will make one check of this assumption by computing the leading higher level and derivative corrections to the tension of the D24-brane.

\subsection{Derivative correction to the tension}

The derivative corrections arise from the appearance of $\tilde{\phi}$ in the interaction terms. The derivatives in the exponential are multiplied by $\alpha^{\prime}$ (which we have set to 1), and so if an $\alpha^{\prime}$ expansion is valid then it is sensible to expand the exponential to linear order and to 
see a small correction to the tension. At this order, this does not in fact introduce higher derivatives into the action, but does introduce $\kappa$ dependence, which one hopes to be an effective expansion parameter. The action is now

$$
S=2 \pi^{2} T_{25} \int d^{26} x\left(\frac{1}{2}[1-8 \kappa \ln (6 \kappa) \phi] \partial_{\mu} \phi \partial^{\mu} \phi-\frac{1}{2} \phi^{2}+2 \kappa \phi^{3}\right) .
$$

When $\phi$ condenses to the local minimum $\phi_{c}$, the coefficient of the kinetic term, which we call $f(\phi) / 2$, becomes

$$
f\left(\phi_{c}\right)=1-8 \kappa \ln (6 \kappa) \phi_{c} \approx-.05 .
$$

The approximate vanishing of the kinetic term at the minimum seems consistent with the conjecture of [10 that tachyon condensation sets all kinetic terms to zero and is related to the observation in [2] that there are no physical poles for the tachyon and transverse component of the $U(1)$ gauge field in the presence of the tachyon condensate. After including higher order corrections, we expect that the vanishing of $f(\phi)$ exactly corresponds with the minimum of the tachyon potential.

We can now solve for the corrected lump solution using

$$
\frac{1}{2} f(\phi)\left(\partial_{\rho} \phi\right)^{2}-V(\phi)=-V\left(\phi_{c}\right)
$$

which yields

$$
\rho=\int_{\phi_{*}}^{\phi} d \phi^{\prime} \frac{\sqrt{f\left(\phi^{\prime}\right)}}{\sqrt{2 V\left(\phi^{\prime}\right)-2 V\left(\phi_{c}\right)}} .
$$

It only makes sense to take the upper limit of integration in the region for which $f(\phi)$ is positive. The boundary of this region is $\phi_{0} \approx .436$, which is slightly less than $\phi_{c}$. One easily finds that this translates into a finite range for $\rho$. Numerically, we find that the vanishing of $f(\phi)$ corresponds to $\rho \approx 3.15$. The corrected lump solution is similar to our previous results for sufficiently small $\rho$, but differs asymptotically. The previous solution only approaches $\phi_{c}$ asymptotically, whereas in the present case the tachyon reaches its vacuum value at finite distance. The two solutions in the small $\rho$ region are displayed in figure 2 .

The computation of the D24-brane tension now proceeds as before, and we find

$$
T_{24}^{\ell}=4 \pi^{2} T_{25} \int_{\phi_{*}}^{\phi_{0}} d \phi^{\prime} \sqrt{1-8 \kappa \ln (6 \kappa) \phi} \sqrt{2 V\left(\phi^{\prime}\right)-2 V\left(\phi_{c}\right)} \approx 4.41 T_{25} .
$$

Including the new term has decreased the tension from $78 \%$ to $70 \%$ of the true value. It is encouraging that the result is a small correction, albeit in the wrong direction.

It would be interesting to examine the results of including more derivative terms in the expansion of the exponential, but that will not be considered here. 


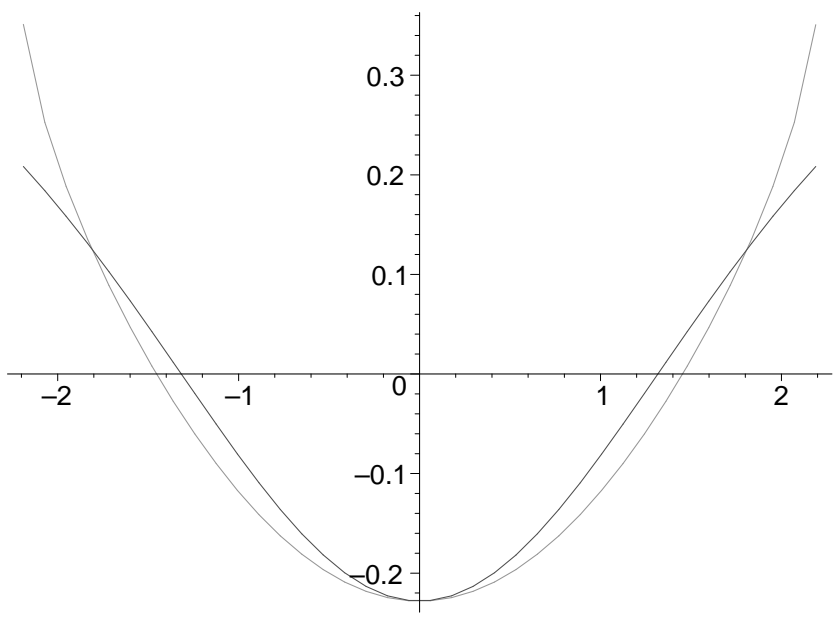

Fig. 2: Comparison of solutions with and without the leading derivative correction. The corrected solution is the one developing a large derivative, and will reach $\phi_{0}$ at finite $\rho$.

\subsection{Higher level corrections}

The tachyon background acts as a source for higher level fields, and so we should examine their effects on the tension. The action is invariant under sign reversal of oddlevel fields, which therefore must appear at least quadratically in the action. Thus it is consistent with the equations of motion to set all odd-level fields to zero [3]. We will study the effect of the following level two fields which are excited by the tachyon background: an auxiliary scalar $\beta_{1}$, a vector $B_{\mu}$, and a symmetric tensor $B_{\mu \nu}$. Including kinetic terms for these fields means that we work to at least level four, and so we should include at least interaction terms quadratic in these fields. In the notation of [2] (see appendix B), we therefore keep $\mathcal{L}^{(2)}+\mathcal{L}^{(4)}$. The interaction terms are an infinite series in derivatives. In keeping with our approach of considering leading corrections, we will truncate $\mathcal{L}^{(2)}$ and $\mathcal{L}^{(4)}$ to lowest order in derivatives, which means replaces tilded fields by untilded fields, and keeping only $\mathcal{L}_{0}^{(4)}$ in the notation of [2]. We stress that this procedure is not necessarily justified; we are considering it in an attempt to understand the systematics of the expansion.

We will take a perturbative approach, consisting of inserting the lowest order tachyon background into the equations of motion of the higher level fields. We will solve for the profiles of the higher level fields numerically, and then numerically evaluate their contribution to the tension. Expanding out the action to the level of accuracy described above, we find that $B_{\mu}$ and the traceless part of $B_{\mu \nu}$ decouple from the other higher level 
fields, while $\beta_{1}$ and the trace of $B_{\mu \nu}$ are mutually coupled. The total action will be written as $S=2 \pi^{2} T_{25} \int d^{26} x \mathcal{L}\left(\beta_{1}, B_{\mu}, B_{\mu \nu}\right)$. We first consider the decoupled fields.

\subsection{Contribution of $B_{\mu}$}

We find

$$
\mathcal{L}\left(B_{\mu}\right)=\frac{1}{2} \partial_{\mu} B_{\nu} \partial^{\mu} B^{\nu}+\frac{1}{2}\left(1+\frac{2^{9}}{3^{4}} \kappa \phi\right) B_{\mu} B^{\mu}-\frac{4}{3} \kappa \phi^{2} \partial_{\mu} B^{\mu}
$$

Our goal is to minimize the energy of $B_{\mu}$ in the presence of the tachyon lump background. Asymptotically, $B_{\mu}$ will vanish, as this minimizes its potential energy for $\phi=\phi_{c}$. Thus we look for the lowest energy solution to the equations of motion following from $\mathcal{L}\left(B_{\mu}\right)$ which vanishes at infinity. It is convenient at this stage to use the approximate form $\phi=\phi_{\ell}$ for the tachyon background. Only the $\rho$ component of $B_{\mu}$ is excited. Solving the equations of motion numerically yields the solution displayed in figure 3 .

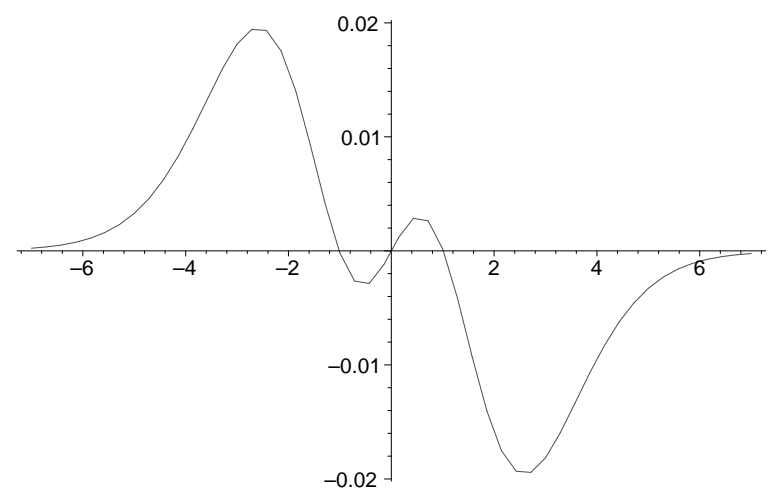

Fig. 3: $\quad B_{\rho}$ profile in the presence of tachyon lump background.

The contribution of $B_{\mu}$ to the lump's tension is then found by integrating the solution

$$
\delta T_{24}^{\ell}=2 \pi^{2} T_{25} \int d \rho \mathcal{L}\left(B_{\mu}\right) \approx-.032 T_{25}
$$

This represents a negligible $.6 \%$ correction to the tension.

\subsection{Contribution of traceless part of $B_{\mu \nu}$}

We first decompose $B_{\mu \nu}$ as

$$
B_{\mu \nu}=\hat{B}_{\mu \nu}+\frac{1}{\sqrt{26}} B \eta_{\mu \nu}
$$


where $\hat{B}_{\mu \nu}$ is traceless. Our definition of $B$ agrees with that of [2]. Now we work out the action of $\hat{B}_{\mu \nu}$ in the presence of the lump. There are contributions both from the components $\hat{B}_{\rho \rho}$ and $\hat{B}_{x x}$, where $x$ denotes coordinates parallel to the lump.

For $\hat{B}_{\rho \rho}$ we find

$$
\mathcal{L}\left(\hat{B}_{\rho \rho}\right)=\frac{1}{2}\left(\partial_{\rho} \hat{B}_{\rho \rho}\right)^{2}+\frac{1}{2}\left(1+\frac{2^{10}}{3^{5}} \kappa \phi\right)\left(\hat{B}_{\rho \rho}\right)^{2}-\frac{2^{4} \sqrt{2}}{3^{2}} \kappa\left[\phi \partial_{\rho}^{2} \phi-\left(\partial_{\rho} \phi\right)^{2}\right] \hat{B}_{\rho \rho}
$$

The profile and tension contribution of $\hat{B}_{\rho \rho}$ are found as above. The profile is displayed in figure 4 .

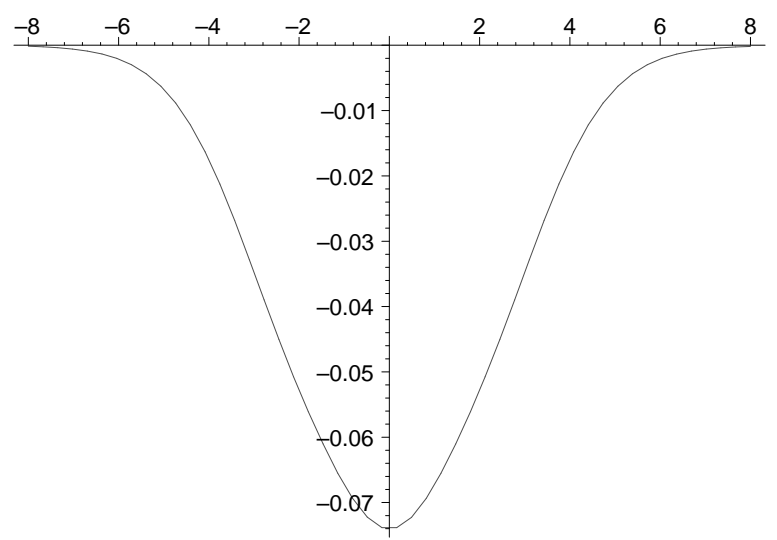

Fig. 4: $\hat{B}_{\rho \rho}$ profile in the presence of tachyon lump background.

For the contribution to the tension we find

$$
\delta T_{24}^{\ell}=2 \pi^{2} T_{25} \int d \rho \mathcal{L}\left(\hat{B}_{\rho \rho}\right) \approx-.27 T_{25},
$$

which corresponds to a $5 \%$ decrease in the tension.

For $\hat{B}_{x x}$ we find for each of the 25 components

$$
\mathcal{L}\left(\hat{B}_{x x}\right)=\frac{1}{2}\left(\partial_{\rho} \hat{B}_{x x}\right)^{2}+\frac{1}{2}\left(1+\frac{2^{10}}{3^{5}} \kappa \phi\right)\left(\hat{B}_{x x}\right)^{2} .
$$

Although there is no term linear in $\hat{B}_{x x}$, this field is excited since in deriving the EulerLagrange equation from $\mathcal{L}\left(\hat{B}_{\mu \nu}\right)$ one must respect the tracelessness of $\hat{B}_{\mu \nu}$, and this produces a source term for $\hat{B}_{x x}$. The profile is displayed in figure 5 .

The contribution of $\hat{B}_{x x}$ to the tension is

$$
\delta T_{24}^{\ell}=25 \cdot 2 \pi^{2} T_{25} \int d \rho \mathcal{L}\left(\hat{B}_{x x}\right) \approx .01 T_{25},
$$

which corresponds to a $.2 \%$ increase. 


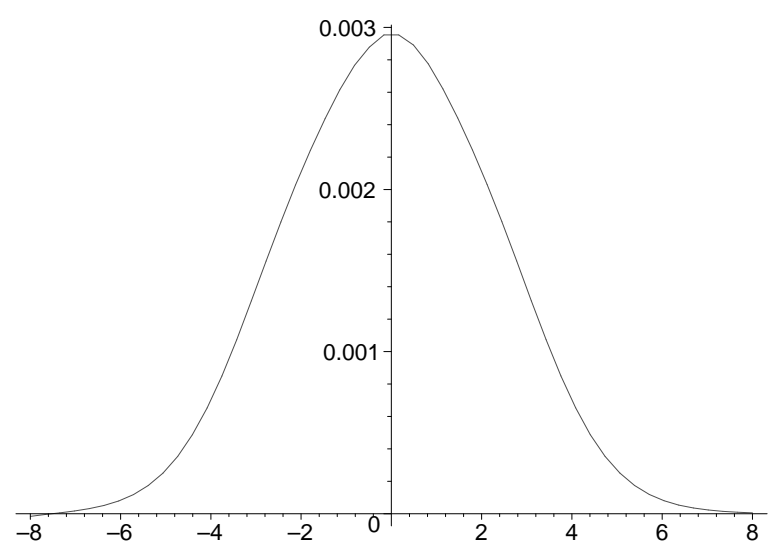

Fig. 5: $\hat{B}_{x x}$ profile in the presence of tachyon lump background.

\subsection{Contribution of $\beta_{1}$ and $B$}

The action for the coupled $\beta_{1}, B$ system is

$$
\begin{aligned}
\mathcal{L}\left(\beta_{1}, B\right) & =\frac{1}{2}\left(\partial_{\rho} B\right)^{2}+\frac{1}{2}\left[1+2\left(\frac{26 \cdot 5^{2}}{3^{5}}+\frac{2^{9}}{3^{5}}\right) \kappa \phi\right] B^{2} \\
& -\left[\frac{\sqrt{2} \cdot \sqrt{26} \cdot 5}{3^{2}} \kappa \phi^{2}+\frac{\sqrt{2} \cdot 2^{4}}{\sqrt{26} \cdot 3^{2}} \kappa\left(\phi \partial_{\rho}^{2} \phi-\left(\partial_{\rho} \phi\right)^{2}\right)\right] B \\
& -\frac{1}{2}\left(\partial_{\rho} \beta_{1}\right)^{2}-\frac{1}{2}\left[1+\frac{2 \cdot 19}{3^{4}} \kappa \phi\right] \beta_{1}^{2}-\frac{2 \cdot 11}{3^{4}} \kappa \phi^{2} \beta_{1} \\
& +\frac{\sqrt{2} \cdot \sqrt{26} \cdot 2 \cdot 5 \cdot 11}{3^{5}} \kappa \phi \beta_{1} B .
\end{aligned}
$$

Note that the auxiliary scalar $\beta_{1}$ has wrong sign kinetic and mass terms. For this reason, the solutions for $\beta_{1}$ and $B$ in the presence of the lump will represent a saddle point of the energy functional (3.13). This makes finding the solution difficult, since the standard Gauss-Seidel algorithm will not converge to the desired solution. In order to obtain a rough estimate of the energy we will proceed as follows. We insert Gaussian wave-functions into the equations of motion following from (3.13), and then vary the height and widths so as to minimize the integrated sum of squared error terms.

We first need to find the boundary conditions at infinity. These are given by the constant field values which extremize the action in the presence of $\phi=\phi_{c}$. We find

$$
B_{c}=.144, \quad \beta_{1}^{c}=-.127 \text {. }
$$

Our Gaussian ansatz is then

$$
B=B_{c}+\alpha_{1} e^{-\alpha_{2} \rho^{2}}, \quad \beta_{1}=\beta_{1}^{c}+\alpha_{3} e^{-\alpha_{4} \rho^{2}} .
$$


Minimizing the error terms in the differential equations leads to the estimate

$$
\alpha_{1} \approx-.2, \quad \alpha_{2} \approx .15, \quad \alpha_{3} \approx 0
$$

Inserting our ansatz back into the action and integrating leads to the following value for the contribution to the tension

$$
\delta T_{24}^{\ell}=2 \pi^{2} T_{25} \int d \rho \mathcal{L}\left(\beta_{1}, B\right) \approx T_{25}
$$

This represents a $20 \%$ increase in the tension.

Given the relatively large magnitude of this correction term, it would clearly be desireable to improve upon the crude estimate given here.

\subsection{Corrected tension}

We can now assemble the various corrections to the tension which we have computed. Our zeroth order computation yielded

$$
T_{24}^{\ell} \approx 4.93 T_{25} \approx .78 T_{24}
$$

Adding the derivative and higher level correction terms gives (only the higher level correction from $\hat{B}_{\rho \rho}, \beta_{1}, B$ are significant)

$$
T_{24}^{\ell}+\delta T_{24}^{\ell} \approx(4.4-.27+1) T_{25} \approx .82 T_{24}
$$

Although the precise value of our result is not significant, we note that we have obtained a small correction in the right direction, which supports the conjecture that the result after including higher order effects will converge to the expected D24-brane tension.

\section{Fluctuation Spectrum of the D24-brane}

In the previous sections we have seen that the level truncation scheme in open string field theory seems to provide a reliable calculation of $D p$-brane tension for large $p$. It is natural to ask whether other features of $D$-branes are also visible in this approximation. In this section we look at the low-lying spectrum of the D24-brane and argue that both the tachyon and $U(1)$ gauge field on the D24-brane are rather accurately described at the lowest non-trivial level. 
We start with the action for the tachyon and gauge field, keeping terms up to level 2. From [2] this is

$$
\begin{aligned}
S= & 2 \pi^{2} T_{25} \int d^{26} x\left[\frac{1}{2} \partial_{\mu} \phi \partial^{\mu} \phi+\frac{1}{2} \partial_{\mu} A_{\nu} \partial^{\mu} A^{\nu}-\frac{1}{2} \phi^{2}\right. \\
& \left.+\kappa\left[2 \phi^{3}+\frac{2^{5}}{3^{2}} A_{\mu} A^{\mu} \phi-\frac{2^{4}}{3^{2}}\left(2 \partial_{\mu} \phi A_{\nu} \partial^{\nu} A^{\mu}-\phi \partial_{\mu} A_{\nu} \partial^{\nu} A^{\mu}-\partial_{\mu} \partial_{\nu} \phi A^{\mu} A^{\nu}\right)\right]\right] .
\end{aligned}
$$

Varying gives the equations of motion:

$$
\begin{aligned}
-\partial^{2} \phi & =\phi-6 \kappa \phi^{2}-\frac{2^{5} \kappa}{3^{2}} A^{\mu} A_{\mu}-\frac{2^{4} \kappa}{3^{2}}\left[3 \partial_{\mu} A_{\nu} \partial^{\nu} A^{\mu}+\partial_{\mu} \partial_{\nu}\left(A^{\mu} A^{\nu}\right)+2 A_{\nu} \partial^{\mu} \partial^{\nu} A_{\mu}\right] \\
-\partial^{2} A_{\nu} & =-\frac{2^{6} \kappa}{3^{2}} \phi A_{\nu}+\frac{2^{5} \kappa}{3^{2}}\left[2 \partial_{\mu} \phi \partial_{\nu} A^{\mu}-2 \partial_{\mu} \partial_{\nu} \phi A^{\mu}-\partial_{\nu} \phi \partial^{\mu} A_{\mu}+\phi \partial_{\mu} \partial_{\nu} A^{\mu}\right] .
\end{aligned}
$$

To study the spectrum of small fluctuations we linearize these equations about the lump solution $\phi_{l}(\rho)$ given in (2.8). Taking $A_{25}=0$, imposing $\partial^{\mu} A_{\mu}=0$, writing

$$
\begin{aligned}
\phi\left(x^{\mu}\right) & =\phi_{l}(\rho)+\psi_{t}(\rho) t\left(x^{m}\right) \\
A_{m}\left(x^{\mu}\right) & =\psi_{a}(\rho) a_{m}\left(x^{n}\right)
\end{aligned}
$$

and substituting into (4.2) gives

$$
\begin{aligned}
\psi_{a} \partial_{n} \partial^{n} a_{m} & =-a_{m}\left(\psi_{a}^{\prime \prime}-\frac{2^{6} \kappa}{3^{2}} \phi_{l} \psi_{a}\right) \\
\psi_{t} \partial_{n} \partial^{n} t & =-t\left(\psi_{t}^{\prime \prime}+\psi_{t}-12 \kappa \phi_{l} \psi_{t}\right)
\end{aligned}
$$

where primes indicate derivatives with respect to $\rho$.

The masses of the fluctuations are thus given by the eigenvalues of the bound states of a one-dimensional Schrodinger equation in a potential proportional to $\phi_{l}(\rho): m_{a}^{2}=$ $\lambda_{a}, m_{t}^{2}=\lambda_{t}-1$ where $\lambda_{a, t}$ are the eigenvalues of

$$
\begin{aligned}
-\psi_{a}^{\prime \prime}+\frac{2^{6} \kappa}{3^{2}} \phi_{l}(\rho) \psi_{a} & =\lambda_{a} \psi_{a} \\
-\psi_{t}^{\prime \prime}+12 \kappa \phi_{l}(\rho) \psi_{t} & =\lambda_{t} \psi_{t}
\end{aligned}
$$

A simple numerical calculation of the minimum eigenvalues of (4.5) gives

$$
m_{t}^{2} \approx-1.3, \quad m_{a}^{2} \approx-.06
$$

in good agreement with the expected values $m_{t}=-1, m_{a}=0$. 
Note that the mechanism responsible for the $U(1)$ gauge field is quite similar to the Randall-Sundrum mechanism for spin 2 zero modes on a domain wall [1]. This should be contrasted with the way that the $U(1)$ gauge field zero mode arises from the space-time point of view for D-branes and NS- IIB fivebranes in the superstring. There the zero mode arises from gauge transformations of R-R and NS-NS tensor fields which are non-trivial in the presence of the brane [12] whereas here the zero mode arises directly from fluctuations of a bulk $U(1)$ gauge field. Such a zero mode would not arise from a general action of the form (4.1) without fine tuning. It seems likely that tachyon condensation in open string theory naturally produces the couplings required to have an exact zero mode.

\section{Conclusions}

We have seen that $D p$-branes can be described as lumps in the open string field theory on a $D 25$-brane, at least for sufficiently large values of $p$. For the $D 24$-brane the tension is close to the expected value and we also find the correct spectrum of low-energy excitations on the brane, namely a tachyon instability and an approximately massless $U(1)$ gauge field. The $U(1)$ gauge field arises by a mechanism similar to the Randall-Sundrum mechanism for gravity [11]. This mechanism might have interesting applications to brane world scenarios.

It would be interesting to extend these results in several directions. $D p$-branes for smaller $p$ should be studied to see whether one can find reliable solutions by including higher order derivative and higher level terms. It is also possible that the level truncation scheme will only be a good approximation for large $p$ and that further understanding will require either an analytic solution or a new approximation scheme. In addition, the higher derivative and higher level terms seem to be conspiring to freeze out the open string degrees of freedom in the vacuum with tachyon condensation, essentially confining open strings. It would be nice to have a better understanding of this phenomenon (see [13], [10] for discussions).

Another interesting direction is suggested by the following argument. Consider a fundamental string ending on a D24-brane. This acts as a source of electric flux on the D24-brane. When the tachyon on the D24-brane condenses the flux should be confined, presumably into a vortex which can be interpreted as a macroscopic string. This suggests that after tachyon condensation it should be possible to directly construct macroscopic fundamental closed strings as vortices in the open string field theory on a D25-brane. 
Finally, it would be interesting to extend the analysis here to the study of both the stable and unstable D-branes of superstring theory using open superstring field theory on unstable $D 9$-branes.

We would like to thank V. Balasubramanian, D. Kutasov and E. Martinec for helpful conversations. This work was supported in part by NSF Grant No. PHY 9901194. 


\section{References}

[1] E. Witten, "Noncommutative Geometry and String Field Theory," Nucl. Phys. B268 (1986) 253.

[2] V .A. Kostelecky and S.Samuel, "On a Nonperturbative Vacuum for the Open Bosonic String," Nucl.Phys. B336 (1990) 263.

[3] A. Sen and B. Zwiebach, "Tachyon Condensation in String Field Theory," hepth/9912249.

[4] A. Sen, "Stable non-BPS bound states of BPS D-branes," JHEP 9808 (1998) 010, hepth/9805019; " $\mathrm{SO}(32)$ spinors of type I and other solitons on brane-antibrane pair," JHEP 9809 (1998) 023, hep-th/9808141; "Type I D-particle and its interactions," JHEP 9810 (1998) 021, hep-th/9809111; "Non-BPS states and branes in string theory," hep-th/9904207, and references therein.

[5] W. Taylor, "D-brane effective field theory from string field theory," hep-th/0001201.

[6] N. Berkovits, "The Tachyon Potential in Open Neveu-Schwarz String Field Theory," hep-th/0001084.

[7] C. G. Callan, I. R. Klebanov, A. W. Ludwig and J.M Maldacena, "Exact solution of a boundary conformal field theory," Nucl.Phys. B422 (1994) 417, hep-th/9402113; J. Polchinski and L. Thorlacius, "Free fermion representation of a boundary conformal field theory,"Phys. Rev. D50 (1994) 622, hep-th/9404008; P.Fendley, H. Saleur and N. P. Warner, "Exact solution of a massless scalar field with a relevant boundary interaction," Nucl. Phys. B430 (1994) 577, hep-th/9406125; A. Recknagel and V. Schomerus, "Boundary deformation theory and moduli spaces of D-branes," Nucl. Phys. B545 (1999) 233, hep-th/9811237.

[8] A. Sen, "Descent Relations Among Bosonic D-branes," Int.J. Mod. Phys. A14 (1999) 4061, hep-th/9902105.

[9] J. A. Harvey, P.Hořava and P. Kraus, "D-Sphalerons and the Topology of String Configuration Space," hep-th/0001143.

[10] A. Sen, "Supersymmetric World-volume Action for Non-BPS D-branes," hep-th/9909062.

[11] L.Randall and R. Sundrum, "An alternative to compactification," Phys.Rev.Lett. 83 (1999) 4690, hep-th/9906064.

[12] C.G.Callan, J.A. Harvey and A.Strominger, "Worldbrane actions for string solitons," Nucl. Phys. B367 (1991) 60; T. Awadi, M. Cederwall, U. Gran, B.E.W. Nilsson and B. Razaznejad, "Goldstone Tensor Modes," JHEP 9902:001 (1999), hep-th/9811145.

[13] P.Yi, "Membranes from five-branes and fundamental strings from Dp branes," Nucl.Phys.B550 (1999) 214, hep-th/9901159. 